\title{
HACIA UN MODELO DE TOMA DE DECISIONES COMPARTIDA PARA EL FINAL DE LA VIDA
}

\section{A MODEL OF SHARED DECISION MAKING AT THE END OF LIFE}

\author{
Javier Barbero', Concepción Prados² y Alicia González' \\ Servicio de Hematología del Hospital Universitario La Paz (Madrid) \\ Servicio de Neumología del Hospital Universitario La Paz. (Madrid) \\ 3 Idi Paz. (Madrid)
}

\section{Resumen}

La toma de decisiones es una práctica diaria de todos los seres humanos. En dicha práctica los criterios que se utilizan se conforman en base a experiencias pasadas, modelos cotidianos o incluso inercias nunca cuestionadas. En el ámbito clínico, sin embargo, debe exigirse un quehacer crítico a la hora de discernir los procedimientos y criterios óptimos para la toma de decisiones, requiriéndose análisis y juicios efectivos y ágiles. A nuestro entender, un planteamiento altamente interesante para abordar decisiones en el ámbito del final de la vida es el de Toma de Decisiones Compartidas (TDC). En el presente texto se pretende, en un primer momento, conceptualizar las variables consideradas más relevantes en dicho modelo, para proponer posteriormente un protocolo que actúe como herramienta facilitadora de la toma de decisiones complicadas a las que pueden verse expuestos los pacientes y sus familiares.

Palabras clave: Toma de decisiones compartidas, cuidados paliativos, final de vida, bioética.

\section{Abstract}

Decision making is a task all human beings have to face up to daily. The underlying basis for this practice is formed and marked by former experiences, every day applied or even automatized models that are never questioned. In the clinical setting, however, a critical reflexion is necessary when deciding procedures and criterias for making decisions, requiring analysis and effective and agile decisions. In our view, a highly interesting approach to tackle decisions in the ambit of the end-of-life is the Shared Decision-Making. In this paper we intend, at first, to conceptualize the most relevant variables according to that model, and then to propose a protocol to act as a facilitating tool for making difficult decisions to which the patients and their families may be exposed to.

Key words. Shared decision making, palliative care, end of life, bioethics.

\section{INTRODUCCIÓN}

La toma de decisiones es una práctica diaria de todos los seres humanos, tanto en el ámbito personal como en el profesional. La inmensa mayor parte de las veces está ajustada a unos criterios debidamente internalizados que, como constancias comportamentales, simplifican la vida. Además, estas decisiones se construyen en cadena, es decir, en medio de un proceso decisional plagado de secuenciales micro-

\section{Correspondencia:}


decisiones. Habitualmente, entre los que contamos con el derecho de recibir un salario a fin de mes -y quizás con la excepción de los fríos días de invierno- uno no se plantea conscientemente qué decisión tomar con respecto a acudir o no acudir esa mañana al trabajo.

Los criterios, en las rutinas diarias, se conforman en base a la experiencia pasa$\mathrm{da}$, los modelos cotidianos o incluso desde las inercias nunca cuestionadas. Es más, la costumbre suele ser vivida como bondad, de ahí la dificultad en replantear determinadas decisiones que rompen con rutinas ya instauradas. Es el denominado realismo moral. Se acaba de entender como bueno aquello que habitualmente existe, lo que racionalmente no deja de ser de un problema, al menos si planteamos la necesidad del progreso.

En el ámbito clínico, no obstante, hay circunstancias que exigen un quehacer crítico a la hora de discernir los procedimientos y los criterios para la toma de decisiones. Ya no sólo por la relevancia habitual de las decisiones -tiene que ver con valores muy sensibles para las personas, como pueden ser la salud, la vida y la calidad de vida- sino también por lo cambiante que son muchas de las variables intervinientes, lo que exige análisis y juicio crítico realmente efectivos y ágiles.

En los escenarios cercanos al final de la vida, las dos decisiones probablemente más difíciles para los profesionales son la limitación del esfuerzo terapéutico(LET) ${ }^{(1)}$ y la transición al cuidado paliativo (CP). Ambas exigen un cambio radical de actitud terapéutica. En las dos supone la renuncia a intentar curar al paciente de sus patologías de base; también en las dos se pretende instaurar de manera predominante la dinámica del cuidar. Ambas son dolorosas, se impone la aceptación de los límites (fronteras) de la medicina y de la limitación (déficit) de la condición humana. La LET suele realizarse con el paciente agudo-crítico cuya crisis ya no se puede resolver positivamente; la transición al CP se suele dar cuando el enfermo con un proceso crónico (neoplasia, enfermedad neurológica grave, etc.) pasa a una situación de terminalidad. En ambos casos el impacto emocional es muy importante y va a condicionar el proceso y, además, la toma de decisiones principal conlleva un cúmulo de nuevas tomas de decisiones añadidas para nada fáciles (retirada o/y no inicio de tratamientos de soporte vital, posibilidad de sedación, planteamiento -si no se ha hecho antes- de elaboración de instrucciones previas, etc., etc.). Como es evidente, en torno a toda decisión clínica aparecen distintos valores en juego, lo que revela la necesidad de tener en cuenta tanto los datos técnicos como los valores éticos que puedan estar en conflicto. Desde esta doble vertiente, técnica y ética, abordaremos el proceso de toma de decisiones.

A nuestro entender, un planteamiento altamente interesante como modelo para este ámbito del final de la vida es el de Toma de Decisiones Compartidas (TDC), que inicialmente intentaremos conceptualizar. En concreto, desarrollaremos las variables referentes a la indicación clínica, la elección del paciente, el tipo formal de decisiones - dilemáticas o problemáticas-, la información, la conocida como comunicación difícil, los estilos de relación clínica y el proceso de deliberación, que incluye tanto variables técnicas, entre ellas las comunicativas, como variables éticas. Posteriormente propondremos un protocolo que integre las variables relevantes previamente comentadas y que actúe como herramienta activa y facilitadora de la toma de decisiones complicadas a las que pueden verse expuestos los pacientes y sus familiares. Para finalizar, expondremos un caso de LET, con un documento de TDC ad hoc y las reflexiones últimas pertinentes. 


\section{MARCO CONCEPTUAL: DELIBERACIÓN, INCERTIDUMBRE Y COUNSELLING}

Se está trabajando mucho en la comprensión del concepto de toma de decisiones compartida $\left(\right.$ TDC) ${ }^{(2)}$, en inglés shared decision making. La expresión TDC fue definida en primer lugar por la President's Comission for the Study of Ethical Problems in Medicine and Biomedical and Behavioral Research, en su informe Making Health Care Decisions $^{(3)}$, que se centró en el consentimiento informado. Este informe se publicó hace casi 30 años (1982) y concibe la TDC como un proceso de mutuo respeto y colaboración:

"Normalmente esto consistirá en discusiones entre el profesional y el paciente que traen el conocimiento, las preocupaciones y la perspectiva de cada uno al proceso de búsqueda de acuerdo en el curso de un tratamiento. Esto significa sencillamente que el médico o cualquier otro profesional invita al paciente a participar en un diálogo en el que el profesional intenta ayudar al paciente a comprender la situación médica y los cursos disponibles de acción, y el paciente expone sus preocupaciones y sus deseos. Esto no implica recitar mecánicamente la abstrusa información médica, pero debería incluir herramientas que aportaran al paciente una mayor comprensión de su condición y una valoración de sus consecuencias (p. 38). El proceso de toma de decisiones compartidas requiere que un clínico no busque solamente comprender las necesidades de cada paciente y desarrollar alternativas razonables para satisfacer esas necesidades, sino también presentar las alternativas de un modo que permita a los pacientes elegir la que ellos prefieren. Para participar en este proceso, el paciente debe establecer un diálogo con el clínico y dejar clara su visión del bienestar".

Ciñéndonos al ámbito médico, para contextualizar la reflexión, podíamos defi- nir la TDC como la interacción médico-paciente en la que hay dos vías de intercambio de información con la participación de ambas partes en la decisión ${ }^{(4,5)}$. Desde las responsabilidades del profesional, al paciente se le provee de información sobre la evidencia de la eficacia del diagnostico o de las opciones de tratamiento y el médico explora los valores personales del paciente relevantes para la decisión particular. En último término, los dos intervinientes toman conjuntamente las decisiones.

Sin embargo, no parece claro cuál es el principio, el mecanismo ${ }^{(6)}$ que media para conseguir estos objetivos de proceso. Por otra parte, el método en sí mismo parece insuficientemente operativo ${ }^{(7)}$. Aparecen muchas definiciones en la literatura, pero inconsistentes entre ellas. Autores como Towle y Godolphin ${ }^{(8)}$ se centran en las competencias comunicativas tanto del médico como del paciente; otros colocan en el médico la responsabilidad de explorar y visualizar la opinión del paciente. Otros cuantos destacan la importancia de proporcionar información al paciente realmente basada en evidencia, mientras que otros priorizan la relevancia del vínculo terapéutico. También en la literatura aparecen otros constructos similares como la toma de decisiones informada ${ }^{(9)}$, la concordancia ${ }^{(10,11)}$, la elección del paciente basada en la evidencia ${ }^{(12,13)}$ y la participación mutua $^{(14)}$.

Makoul y Clayman $^{(7)}$ realizan una interesante revisión de las variables conceptuales desde las que se define en la literatura el concepto de TDC. Recogen aquellas que aparecen en más de un 10\% de los artículos consultados. En la Tabla 1 las señalamos. Llama la atención que sólo hay dos variables (valores / preferencias del paciente $y$ opciones) que superan el dintel del $50 \%$.

También es llamativo que ninguno de los instrumentos disponibles tome en cuenta el carácter interactivo de la TDC. 


\section{Tabla 1. Categorías mencionadas en al menos el $10 \%$ de los artículos en los que se define la TDC, elaborado por G. Makoul y M. Clayman ${ }^{(7)}$}

\begin{tabular}{|l|l|}
\hline $61,7 \%$ & Valores del paciente / preferencias \\
\hline $50,9 \%$ & Opciones \\
\hline $46,0 \%$ & Colaboración \\
\hline $37,3 \%$ & Participación del paciente \\
\hline $36,6 \%$ & Educación del paciente \\
\hline $35,4 \%$ & Beneficios / riesgos (pros y contras) \\
\hline $31,7 \%$ & Deliberación / negociación \\
\hline $30,4 \%$ & Conocimiento del doctor / recomendaciones \\
\hline $29,2 \%$ & Acuerdo mutuo \\
\hline $26,7 \%$ & Procesos / fases \\
\hline $23,6 \%$ & Término medio \\
\hline $23,0 \%$ & Intercambio de información \\
\hline $18.0 \%$ & Hacer o explícitamente aplazar la decisión \\
\hline $16,8 \%$ & Evidencia presente \\
\hline $13.0 \%$ & Roles definidos (deseo de involucración) \\
\hline $11,8 \%$ & Información imparcial \\
\hline $11,8 \%$ & Verificar / clarificar comprensión \\
\hline $11,2 \%$ & Flexibilidad / enfoque individualizado \\
\hline $10,6 \%$ & Respeto mutuo \\
\hline
\end{tabular}

$\mathrm{Ni}$ el rendimiento de las habilidades de cualquiera de los participantes, ni la percepción posterior del paciente de su participación nos dan una estimación adecuada de la extensión con la cual la comunicación ha sido realmente compartida entre las partes. En resumen, el paradigma prometedor de la TDC parece necesitar considerar su carácter interpersonal y proporcionar explicaciones acerca de su modo de funcionamiento.

Nosotros proponemos un modelo de TDC que puede ser fundamentado y de- sarrollado a partir de la deliberación, concepto capturado desde la bioética occidental y contemporánea sobre todo a partir de los desarrollos del profesor Diego Gracia $^{(15)}$ y a partir también de la sistematización como modelo de relación terapéutica realizada por Linda y Ezequiel Emanuel $^{(16)}$. Este modelo viene complementado por la reflexión sobre el manejo de la condición de incertidumbre en la decisión clínica y por la necesidad de desarrollar estrategias comunicativas como el counselling ${ }^{(17)}$, facilitadoras claves del pro- 
ceso de toma de decisiones. No hay que olvidar que la TDC sirve a un propósito comunicativo y terapéutico y por tanto ha de seguir un proceso formal, abstraído de las situaciones concretas, como puede ser el proceso deliberativo, y una estrategia de comunicación interpersonal mediadora de la transferencia de contenidos y valores entre las partes.

Una breve consideración en torno al papel de la incertidumbre. En primer lugar, no perder la perspectiva de que es un aspecto clave de la comunicación del riesgo. La incertidumbre es inherente al pronóstico individual, a la capacidad de un tratamiento en mejorar la salud del paciente, a la evaluación de la comprensión del paciente de las explicaciones dadas, etc., etc. Además la incertidumbre difícilmente puede ser resuelta en una conversación, pues suele estar cargada no sólo de datos técnicos promedio, sino también de la dificultad de abordar la subjetividad y la personalización. Independientemente de la decisión particular de que se trate (tratamiento de una recaída, cambio de actitud a LET, etc.), podemos entender la incertidumbre como el contenido central de la comunicación decisional. En realidad, el intercambio de información implícitamente se refiere a una estrategia para comunicar incertidumbres. En el ámbito de la oncología, Kasper et al. (2008)(18) han investigado acerca de las temáticas de incertidumbre en la persona con cáncer (ver Tabla 2). Ciertamente, no son pocas. A modo de ejemplo, la integración social (la fiabilidad de los apoyos sociales de cara al afrontamiento de la enfermedad), el diagnóstico y el pronóstico, la necesidad de descifrar la información y conducta del médico, la repercusión en otros ámbitos vitales como puede ser el laboral, la atribución causal de la aparición de la enfermedad, el compromiso a adquirir en las decisiones (no querer parecer muy pesado, etc.), la valoración sobre la competencia del equipo sanitario, la eficacia atribuida a los tratamientos, etc. Todo ello son escenarios de incertidumbre.

Ya centrándonos en la TDC, si partimos que se basa en dos elementos, a saber, el carácter de doble dirección de la transferencia y la igualdad en la relación interpersonal, parece claro que metodológicamente la TDC tendrá que especificar qué estrategia se ha de utilizar para comunicar incertidumbre.

\section{CUESTIONES CLAVES}

\section{La cuestión de la indicación clínica}

La indicación clínica no depende únicamente del nivel de conocimientos del profesional. Los conocimientos son condición necesaria pero no suficiente para una correcta toma de decisiones en este ámbito. Como bien nos dice el Harrison ${ }^{(19)}$, manual clásico de la medicina, existen determinadas condiciones necesarias para la toma de decisiones. En primer lugar, una habilidad que permita extraer a partir de un cúmulo de signos físicos contradictorios y de los datos del laboratorio sólo aquéllos datos que son de importancia crucial a la hora de saber si es necesario "tratar u observar" un caso difícil; es decir, discernir qué datos son relevantes y cuáles pertenecen al espacio de lo indiferente. En segundo lugar, la necesidad de una cierta capacidad para determinar cuándo un indicio clínico merece estudiarse o bien es una "falsa alarma"; dicho de otro modo, de los datos teóricamente relevantes cuáles han de ser trabajados y cuáles abandonados; en último término, se plantea la necesidad de herramientas para valorar si el tratamiento propuesto entraña para el paciente un riesgo mayor que la propia enfermedad; en otras palabras, una adecuada valoración de riesgos.

Los profesionales tenemos una responsabilidad crucial en realizar indicaciones 
Tabla 2. Categorías de incertidumbre, definiciones y afirmaciones de ejemplos de entrevistas a gente que sufre cáncer. Adaptado de Kasper et al. (18)

\begin{tabular}{|c|c|c|c|}
\hline $\begin{array}{l}\text { Incerti- } \\
\text { dumbre }\end{array}$ & $\begin{array}{c}\text { con respecto } \\
\text { a... }\end{array}$ & $\begin{array}{c}\text { Esta categoría incluye } \\
\text { incertidumbres relacionadas } \\
\text { con... }\end{array}$ & Afirmación típica \\
\hline 1 & $\begin{array}{l}\text { Integración } \\
\text { social }\end{array}$ & $\begin{array}{l}\text {... la fiabilidad de las } \\
\text { relaciones sociales de } \\
\text { cara a las dinámicas de la } \\
\text { enfermedad }\end{array}$ & $\begin{array}{l}\text { A menudo pienso en } \\
\text { mi esposa, me pregunto } \\
\text { cuánto tiempo podrá } \\
\text { aguantar. }\end{array}$ \\
\hline 2 & $\begin{array}{l}\text { Diagnóstico y } \\
\text { pronóstico }\end{array}$ & $\begin{array}{l}\text { El estado normal de la } \\
\text { enfermedad y su curso futuro. }\end{array}$ & $\begin{array}{l}\text { No sé cómo interpretar } \\
\text { esta clase de dolor que } \\
\text { nunca sentí antes, ¿sabes? }\end{array}$ \\
\hline 3 & $\begin{array}{l}\text { Descifrar } \\
\text { información }\end{array}$ & $\begin{array}{l}\text { La interpretación de la } \\
\text { conducta del equipo médico } \\
\text { y otras informaciones } \\
\text { recibidas por el paciente. }\end{array}$ & $\begin{array}{l}\text { Mi médico me habló del } \\
\text { procedimiento diagnóstico } \\
\text { adicional. Pienso que él } \\
\text { ya sabía lo del tumor, } \\
\text { pero no quería decírmelo. }\end{array}$ \\
\hline 4 & $\begin{array}{l}\text { El dominio de } \\
\text { requerimientos }\end{array}$ & $\begin{array}{l}\text { La habilidad de afrontar la } \\
\text { enfermedad relacionada con } \\
\text { los cambios vitales. }\end{array}$ & $\begin{array}{l}\text { Entonces, qué pasa con lo } \\
\text { de mi trabajo... }\end{array}$ \\
\hline 5 & $\begin{array}{l}\text { Atribución } \\
\text { causal }\end{array}$ & $\begin{array}{l}\text { Integración cognitiva de ser } \\
\text { afectado por una enfermedad } \\
\text { crónica. }\end{array}$ & $\begin{array}{l}\text { Quizás hice algo } \\
\text { equivocado en mi vida. } \\
\text { Quizás Dios... bien, no } \\
\text { sé... }\end{array}$ \\
\hline 6 & $\begin{array}{l}\text { Grado propio } \\
\text { preferido de } \\
\text { compromiso }\end{array}$ & $\begin{array}{l}\text { Hasta dónde el paciente } \\
\text { tiene la voluntad de jugar un } \\
\text { papel activo en la interacción } \\
\text { médico-paciente. }\end{array}$ & $\begin{array}{l}\text { Me preocupa si vuelvo } \\
\text { loco a mi médico } \\
\text { expresándole todas mis } \\
\text { dudas... }\end{array}$ \\
\hline 7 & $\begin{array}{l}\text { Confiabilidad } \\
\text { del médico }\end{array}$ & $\begin{array}{l}\text { Competencias profesionales } \\
\text { y personales del equipo } \\
\text { médico. }\end{array}$ & $\begin{array}{l}\text { Yo creo que él está al día. } \\
\text { Al menos así lo espero... }\end{array}$ \\
\hline 8 & Tratamiento & $\begin{array}{l}\text { La eficacia de un tratamiento } \\
\text { tanto como de otras } \\
\text { actividades de soporte. }\end{array}$ & $\begin{array}{l}\text { Al principio, yo } \\
\text { leía algunos libros } \\
\text { de medicina china, } \\
\text { acupuntura, etc. Pero } \\
\text { afortunadamente la } \\
\text { quimio no fue tan dura. }\end{array}$ \\
\hline
\end{tabular}


diagnósticas y terapéuticas ajustadas a criterios de buena práctica clínica, es decir, la decisión de la indicación, no indicación o contraindicación de un determinado procedimiento (medicamentoso, psicoterapéutico, quirúrgico, etc...) ha de estar fundamentada en la denominada como medicina - $\mathrm{u}$ otras disciplinas- basada en la evidencia, con los mejores niveles de certidumbre posibles. Éste es el terreno de los profesionales y, en torno a él, es en el que aparece el principio de no-maleficencia. Es decir, nuestra gran responsabilidad en la decisión es no hacer nada que esté contraindicado, proponer intervenciones indicadas y tolerar -si no hay otra alternativa y no transgrede significativamente el principio de justicia- las decisiones que pertenecen a la no-indicación.

\section{La elección del paciente}

El profesional, en el proceso de toma de decisiones, ha de ser celoso del terreno de la indicación, es su responsabilidad, pero obviamente, no puede ser el único criterio utilizado. Un paciente puede rechazar un tratamiento indicado y solicitar uno no-indicado (aunque no esté contraindicado), si hay otras variables de tipo biográfico relevantes que piden ser tenidas en cuenta. Por ejemplo, un paciente en el final de su vida, que padece un dolor incoercible, realmente refractario, y que prefiere no ser sedado, aunque esté indicado, por su deseo de recibir a y despedirse de su única hija que vive en Australia y que viene a verle.

El profesional indica, pero el paciente elige -o al menos así debería ser-, y lo hace habitualmente dentro de la gama de alternativas técnicas que están indicadas. Incluso puede rechazar el tratamiento, como bien refleja la Ley $41 / 2002^{(20)}$, de Autonomía del Paciente.

Hemos puesto a propósito el término "elección" y no el de "decisión" en el encabezamiento de este apartado. Distinguimos, en aras de facilitar la reflexión, entre el ámbito clínico y pretendidamente objetivo de la indicación (más inclinado hacia el lado de la protección y promoción de la salud biológica del sujeto) y el ámbito personal y pretendidamente subjetivo -sin ningún matiz peyorativo en ello- de la elección (más tendente hacia el lado de la protección y la promoción de la salud biográfica de la persona). Tanto la indicación como la elección forman parte intrínseca de la decisión. No obstante, seguiremos manteniendo el término decisión indistintamente, pues es el contexto el que aporta los matices, en un sentido u otro.

En esta línea, para que la decisión, (es decir, para que la elección...) sea claramente autónoma, se han de dar estas cuatro condiciones:

a) La decisión ha de ser informada, es decir, el paciente ha de conocer el contenido de las alternativas, los efectos y riesgos esperados de cada una de ellas en sus distintas temporalidades, incluida la alternativa del no-tratamiento, etc.

b) La decisión ha de ser voluntaria; dicho de otro modo: ni coaccionada ni manipulada. La coacción aparece cuando se vierte una amenaza, sea explícita o implícita, y la manipulación puede darse tanto por distorsión cognitiva (presentación no adecuada de los datos, por ejemplo) como por el conocido como chantaje emocional.

c) La decisión ha de realizarse por una persona mentalmente competente $^{(21)}$, tanto desde el punto de vista cognitivo (capacidad de valorar alternativas, de comparar las consecuencias previstas, de justificar la decisión...), como desde el emocional (no en presencia de una 
depresión importante o de un ataque de pánico, etc.).

d) La decisión ha de ser auténtica, es decir, al menos no ha de entrar en contradicción frontal con el mundo de valores de la persona. Es un criterio de falsación. No es necesario que justifique la correlación de la decisión con sus valores, pero sí al menos que no entre en clara contradicción con los mismos. Aquí va a ser crucial valorar los mecanismos de afrontamiento (o mecanismos de defensa) de la persona, pues determinadas estrategias, como puede ser la negación, si son desadaptativas, pueden hacer una decisión inauténtica.

Los pacientes suelen aportar al proceso de toma de decisiones tres elementos importantes: información, expectativas y preferencias y en la transacción de los tres aparecerá esencialmente su mundo de valores; sobre el contenido y el nivel de estos tres elementos habremos de establecer un adecuado proceso de deliberación. Nuestra formación clínica nos facilita la exploración de la información que trae el paciente sobre su estado físico y sobre su bienestar o malestar corporal, a partir de sus síntomas; no obstante, la exploración de expectativas y preferencias es algo más complejo, así como el nivel de información que desea el paciente. Todo ello, como luego veremos, tiene que ver con la denominada "comunicación difícil".

\section{La información}

Vamos a pararnos, con un cierto detenimiento, en la cuestión a veces tan conflictiva de la información a los pacientes, porque para tomar decisiones, el paciente tiene que poder ejercer el conocido como "derecho a la verdad(22)". Pero ¿qué es eso de "la verdad"? Indudablemente estamos ante una palabra polisémica a la que debemos acercarnos desde distintos marcos conceptuales. Sin aclarar estas cuestiones previas difícilmente podremos ponernos de acuerdo y llegar a algún consenso. Partimos de la hipótesis de que estamos, al menos, delante de tres acepciones:

a) La primera podríamos denominarla la verdad objetiva. Se trata de adecuar la palabra a la realidad material de los hechos. Esta acepción proviene del mundo de la Grecia Clásica, fundamentalmente de los platónicos, que nos hablan de la existencia de un mundo de la luz y un mundo de las sombras. La tarea del "filósofo", es decir, del "amigo de la sabiduría" es desvelar lo oscuro y llevarlo a la luz, pasar de la ignorancia a la verdad objetiva. A partir de ahí, por medio de convencionalismos, daremos nombre a la verdad que iluminamos, de una manera u otra. Por ejemplo, las enfermeras convienen que una puntuación $<14$ en la escala de Norton objetivamente implica un determinado riesgo de úlceras por presión para el paciente. Es una verdad convencional, pero "objetiva". En el caso de los médicos, después de realizar un diagnóstico diferencial, se puede determinar que una tumoración en grado " $x$ " de Dukes supone un cáncer con un pronóstico estimado de -pongamos- menor de tres meses. Esta es la verdad...

Para los griegos este tipo de verdad no estaba al alcance de todos los humanos, sino solamente a disposición de unos cuantos. En el terreno social, sólo será el rey filósofo el que pueda determinar qué es verdadero en la polis y en el terreno biológico, sólo será el médico el único capaz de conocer la verdad en un organismo humano. Esto, como luego veremos, va a tener consecuencias muy concretas. No olvidemos que la información es una fuente de poder.

Actualmente, en el terreno sanitario, muchos profesionales, en sentido riguroso, sólo admitirían como verdadero lo que se 
ha probado experimentalmente, en estudios controlados, doble ciego, prospectivos, con muestras randomizadas y -icómo no!-, publicado en revistas científicas de alto prestigio. Es el lenguaje objetivo de los datos estadísticos. Si no tanto de la estadística inferencial, sí al menos de la estadística descriptiva.

b) La segunda acepción del término verdad tiene matices estrictamente morales. Resulta curioso observar cómo existe un deslizamiento conceptual de los valores lógicos (lo correcto o incorrecto), a estimaciones de verdadero / falso y de éstas a valoraciones morales de bueno / malo. Puede parecer extraño, pero las valoraciones de correcto, bello y verdadero suelen correlacionar con la de bueno, mientras que las de incorrecto, feo y falso con el valor moral de maldad. En algunos códigos morales antiguos se llega a admitir que, ante la duda de dos sospechosos de un delito, tiene más probabilidades de ser el delincuente el "peor encarado". Estas valoraciones se pueden trasladar a nuestro contexto actual. Una persona sucia (Ilamativo el doble matiz), incorrectamente vestida, desastrada... a las 2 de la madrugada levanta en nosotros sospechas de persona peligrosa de la que conviene alejarse. De ella se espera una conducta inmoral. De igual modo que un drogodependiente del que se suele decir que sistemáticamente miente, de esta afirmación sacamos la conclusión no sólo de que sea incorrecto lo que nos dice -es decir, no ajustado a la realidad-, sino que tiene una catadura moral sospechosa. En la relación clínica con los pacientes se pueden esconder, de forma no necesariamente consciente, puntos de vista similares. Cuando aparece conflicto entre los pacientes y los profesionales, se tiende a pensar que aquéllos, en función de su deterioro y de sus intereses particulares realizan planteamientos incorrectos $y$, por tanto, falsos, mientras que los profesionales, en función de su capacidad científica y de sus intereses generalizables plantearán posturas correctas y, por tanto, verdaderas.

c) La tercera acepción está muy vinculada a la anterior y viene a referir la verdad como un concepto relacional desde el doble matiz de fidelidad y confianza. Esta tercera acepción proviene de una raíz histórica tan importante para nosotros como es el mundo semita, en su tradición judeocristiana. Revisando algunos textos sagrados de los judíos (Antiguo Testamento) o cristianos (el Nuevo Testamento) podemos encontrar expresiones tan sorprendentes como "la verdad os hará libres" (Jn 8, 32). ¿Se refiere a que el conocimiento, la información sobre la realidad da poder y éste te da más posibilidades de ser libre? No parece. Según los exegetas, hace referencia a la verdad como un tipo de vínculo con Dios basado en la confianza y la fidelidad, siendo esto lo que realmente libera al hombre.

También resulta curioso observar algunas expresiones castellanas, probablemente con un origen semítico en su construcción. Cuando decimos que "Pedro es un amigo de verdad" nos estamos refiriendo básicamente a que me puedo fiar a fondo de él, que me merece toda la confianza, no al hecho de que me vaya a desvelar el lado oscuro de su vida o que yo pueda hacer lo mismo. Eso sí, normalmente desde la relación de confianza y fidelidad probablemente también surgirá compartir eso que está oculto, pero no es condición indispensable. De hecho, en la vida diaria no solemos exigir a los amigos, en una relación madura, conocer toda su vida, sino fidelidad y confianza. En la misma línea, contamos con alguna que otra curiosa expresión como la de "ése... es un falso". ¿Qué significa, que no cuenta nada, que es tímido, que oculta necesariamente información? Más bien, que es una persona que no me inspira ninguna confianza $y$ de la que creo que debo desconfiar, con la que no puedo mantener una verdadera 
relación. En un planteamiento de TDC difícilmente se podrá desarrollar si la relación, si el vínculo terapéutico no está basado en la fidelidad y en la confianza.

En el ámbito sanitario, ante situaciones de diagnóstico y/o pronóstico grave tradicionalmente se suelen aducir distintos $\operatorname{argumentos}^{(23,24)}$ para no decir la verdad a los pacientes:

$1^{\circ}$ "Engaño benevolente": "lo que uno no conoce no puede herirle y puede ayudarle". Informar sólo añadiría un plus de ansiedad al paciente y esto es evitable. Es un planteamiento puramente consecuencialista, aunque desconocedor de los resultados de la literatura ${ }^{1}$.

$2^{\circ}$ Los profesionales no tienen capacidad para conocer la "verdad completa" $y$, aunque lo fueran, muchos pacientes no serían capaces de comprender el objetivo y las implicaciones de la información.

$3^{\circ}$ Los enfermos con patologías graves y/o clínica de deterioro, incluso cuando dicen que quieren saber, en realidad prefieren no saber.

Que el desvelar la información, en principio, daña más que beneficia ha sido desmentido por la investigación ${ }^{(25)}$. Es más, es conocido que los pacientes que conocen su diagnóstico, no sólo no pierden la esperanza, sino que muestran más confianza en el cuidado que reciben.

El acceso a la verdad es un derecho de todos los pacientes. Sencillamente porque uno tiene derecho a decidir, con apoyo y con conocimiento de causa, sobre aspectos tan importantes de su vida como puede ser su proceso de salud/enfermedad, de vida/muerte. ¿Pero qué significa eso de ser fieles a la verdad del paciente? Por lo menos, siguiendo las dos acepciones fundamentales de nuestra tradición, que se puedan cumplir estas dos condiciones:

ofrecerle la información objetiva de todo lo que quiera saber y sólo lo que quiera saber acerca de su "ver- dad objetiva", material; esta verdad la averiguaremos explorando -con técnicas comunicativas pertinentes- el mundo vital de la persona, para evitar tanto el pacto de silencio, como el "encarnizamiento informativo".

ofertarle una garantía de soporte adecuada ante la fragilidad que supone la enfermedad y la fase terminal.

La información y la fidelidad relacional se construyen, entonces, mediante un proceso, no en sucesos puntuales, donde la clave, más que en el nivel de información, se encuentra en el nivel de comunicación afectiva y efectiva que se logra. Desde esta perspectiva, podremos considerar la información válida como:

] integradora (tiene en cuenta la realidad paciente / familia / contexto sociocultural)

$\square$ dinámica y de doble dirección

$\checkmark$ secuencial, atenta a la evolución de los intervinientes. Importancia del concepto "economía" de la verdad o del proceso "información a plazos".

$\square$ finalista, pues puede ser un medio idóneo para regulación emocional, autonomía en las decisiones, adhesión al tratamiento, adaptación a la situación, etc.

\section{Comunicación difícil}

Comunicar malas o micro-malas ${ }^{(26)}$ noticias, responder a preguntas difíciles, abordar la cuestión del pacto de silencio, tan típico de los CP, incorporar el apoyo emocional al proceso informativo, acoger las reacciones ansiosas de pacientes y familiares, etc., todos ellos son ejemplos de la denominada como comunicación difícil. No podemos plantearnos una adecua- 
da toma de decisiones si no contamos con herramientas comunicativas para afrontar estas situaciones. ¿Qué decisión se puede tomar tras la comunicación de una mala noticia si no conocemos las pautas de manejo de reacciones tan habituales como el bloqueo o el estallido emocional? De todos es conocida la influencia que puede tener una emoción como el miedo, por poner sólo un ejemplo, en cualquier proceso de toma de decisiones y, más aún, cuando incide en algo tan radicalmente importante como es la salud o la calidad de vida.

La participación en el proceso de toma de decisiones ha de ser, a nuestro entender, claramente interdisciplinar. El sistema abandona a los médicos a su suerte, sin darles las herramientas necesarias para el manejo de la comunicación difícil. En primer lugar los pacientes, pero también los profesionales de la medicina tienen el derecho a poder contar con profesionales expertos en estas lides, como son los psicólogos $^{(27)}$, que faciliten el complejo proceso de toma de decisiones, que incluye tanto acoger la ansiedad que le acompaña como prevenir e intervenir frente a la posible aparición del sentimiento de culpa en los intervinientes.

\section{El tipo de decisiones: dilemáticas o problemáticas}

Escribe Diego Gracia ${ }^{(28)}$ que existen dos tipos de aproximación fundamental a los problemas éticos, y también al uso de las metodologías para darles respuesta, que pueden llamarse "dilemático" y "problemático". La aproximación dilemática generalmente elige entre dos posibilidades diferentes, y utiliza una metodología "decisionista" basada en la creencia de que los problemas éticos tienen siempre y necesariamente una respuesta y que ésta es siempre única. Este método de decisión acaba convirtiendo los problemas éticos en problemas técnicos no-éticos, y es por eso muy del gusto de los utilitaristas y también de los deontologistas duros (los extremos acaban tocándose).

Los partidarios de la aproximación problemática subrayan la importancia del proceso más que de la meta. Se trata de una aproximación "deliberacionista". Su objetivo principal es el procedimiento, no la conclusión, entre otras razones porque no es evidente que todos los problemas morales tengan siempre solución, y en los casos en que sí, tampoco es evidente que sólo sea una y la misma para todos. Los problemas son cuestiones que no sabemos si seremos capaces de resolver, ni cómo lo haremos. La solución no está presente desde el comienzo y, por tanto, no puede ser la decisión entre dos posibles respuestas, sino la creación, la producción de una respuesta propia y adecuada que pueda propiciarse desde un proceso de TDC, porque tendrá en cuenta los intereses y valores de todas las partes en conflicto.

\section{La relación clínica}

Ezekiel y Linda Emanuel ${ }^{(16)}$ han Ilegado a distinguir cuatro modelos de relación clínica, basándose en cómo se tratan en cada uno de ellos los valores y la autonomía del paciente, las obligaciones y el rol del médico. Concretamente, se refieren a los modelos paternalista, informativo, interpretativo y deliberativo. Ellos defienden este último, aunque afirman que "... diferentes modelos pueden ser apropiados cuando varían las circunstancias clínicas. De hecho, los cuatro modelos pueden servir de guía a médicos y pacientes en diferentes momentos. Sin embargo, es importante determinar un modelo que pueda ser asumido por todas las partes, una base de referencia; los otros modelos podrán ser usados excepcionalmente, pero requerirán una justificación basada en las circunstancias concretas de la situación médica". 
Los procesos de TDC son claramente compatibles con el modelo de deliberación, dado que éste admite que los valores pueden evolucionar y estar abiertos a discusión cuando entran en conflicto (no se trata sólo de rescatar los valores fijos de un paciente, escondidos en la hojarasca de las emociones que conlleva la enfermedad), que los interlocutores son reconocidos como sujetos y no sólo como provisores o receptores de cuidados y que se puede llegar a un compromiso entre los criterios de indicación y los de elección a partir de la escucha atenta y empática de cada una de las partes. Desde esta perspectiva, las obligaciones del profesional están en estructurar la deliberación, informar al paciente de las alternativas técnicas disponibles, ayudarle a visibilizar los valores que hay en juego en la decisión, las posibilidades que ofrece su autonomía moral, así como las limitaciones (sobre todo desde el ámbito de la no-maleficencia y de la justicia) y, en último término, realizar la intervención elegida por él.

Desde el punto de vista de la TDC el modelo deliberativo ofrece particularidades muy interesantes. El profesional se sitúa en el plano del acompañamiento de un paciente en un proceso en el que el protagonista, por así decirlo, es el paciente y el coprotagonista es el profesional, pues éste decide no mirar hacia otro lado ni abandonar a su suerte a ese paciente que, desde su autonomía moral, nos puede estar pidiendo que no le dejemos solo en un difícil y a veces doloroso camino. Para el que en último término elige -el paciente- va a ser mucho más fácil hacerlo si se sabe realmente acompañado por un interlocutor para él tan importante como el profesional que domina el ámbito de la indicación técnica.

Para un proceso de TDC, la autonomía del paciente, desde la deliberación, se concibe como un claro autodesarrollo moral, lo que permite incorporar claramente otros valores añadidos al de la curación o supervivencia, es decir, al de la vida biológica, facilitando la posibilidad de estar abiertos a nuevas jerarquías y preferencias que incluyan aspectos tan subjetivos pero tan transcendentales como el de la calidad de vida; por otro lado, la deliberación, huyendo de la perspectiva dilemática, también permite aceptar la perspectiva de proceso y, más en concreto, de proceso de adaptación, lo que también nos facilita, por poner un ejemplo muy común al final de la vida, contemplar el derecho al miedo y a que el proceso de TDC no siga un curso lineal.

\section{El proceso de deliberación moral}

Posiblemente, el amable lector, si no le hemos aburrido suficientemente con este texto y ha llegado hasta el presente párrafo, se habrá preguntado si tras un buscado proceso de TDC se encuentra el objetivo de Ilegar a acuerdos. Es más, algunos, de vocación más autonomista, hasta podrían afirmar que esto no puede ser un objetivo sino, en todo caso, una consecuencia bien recibida pero no necesariamente buscada, porque aquí lo realmente importante es que el paciente elija en función de sus valores (expresados habitualmente en forma de preferencias o expectativas). En la misma línea, muchos se preguntarán si el respeto a la autonomía moral del paciente nos obliga solamente a exponer alternativas, suficientemente bien informadas, pero no a proponer determinados itinerarios asistenciales, precisamente en aras del respeto debido a los pacientes. Dicho de otro modo, no sólo se trata de -obviamente- no coaccionar y no manipular, sino también de no proponer para no condicionar en función de nuestros deseos o particularidades.

Nuestra posición es un tanto distinta. A nuestro entender, hay un terreno, el de la intersubjetividad, que trata de determinados valores ampliamente compartidos con 
la comunidad moral a la que permanecemos. Por poner un ejemplo, la mayor parte de las personas prefieren no padecer dolor físico que sufrirlo y, por tanto, es claramente legítimo proponer de manera preventiva una determinada analgesia siguiendo protocolos consensuados por los especialistas. Por tanto, tengo la responsabilidad de proponer y no sólo exponer el protocolo y mi objetivo será llegar a acuerdos con el paciente en esta materia, porque me parece muy central desde una perspectiva terapéutica. No obstante, el proceso (de deliberación) compartido acaba teniendo sentido aunque el resultado no haya sido la consecución de un acuerdo.

Conviene no olvidar que, como se afirma en una publicación de la Organización Médica Colegial precisamente en torno a la ética en $\mathrm{CP}^{(29)}$, "el objetivo de la deliberación es la toma de decisiones prudentes o razonables. No se trata de que todo el mundo adopte la misma decisión ante un caso concreto, sino que todos, tomen la que tomen, Ileguen a decisiones prudentes. Se trata de incrementar la prudencia de las decisiones elegidas, aunque éstas no coincidan entre sí. El hecho de que las decisiones varíen entre las personas no es problema grave, siempre y cuando todas sean prudentes. La deliberación es un procedimiento que intenta enriquecer el análisis a fin de incrementar la prudencia".

Llegar a acuerdos no siempre es fácil. ¿Cuáles son las condiciones que nos permiten poder llegar a acuerdos, en un ámbito tan complejo como el de la relación clínica en escenarios de dificultad?

a) En primer lugar, un lenguaje más unívoco. El lenguaje clínico puede parecernos poco equívoco, sin embargo, forma parte de un universo comunicativo muy restringido, el de los profesionales, al que no suele tener acceso en detalle la población general. Y no digamos con respecto al lenguaje ético en torno a los valores. Cuando un paciente dice que "no es justo que tenga que esperar 20 minutos a una consulta", probablemente funciona con un sentido de lo que es la justicia muy distinto al que habitualmente manejamos en los hospitales, por ejemplo. Difícilmente podemos llegar a acuerdos sin un mínimo de lenguaje común, de ahí la necesidad de un esfuerzo comunicativo por nuestra parte.

b) Una fundamentación racional ${ }^{30}$ (no emocional ni creencial) de las valoraciones. Las emociones suelen ser malas consejeras para la toma de decisiones (sobre todo -pero no sólo- la angustia y el miedo); las creencias, por otra parte, son importantes para las decisiones individuales, pero inoperantes si hay interés real en llegar a algún tipo de acuerdo en una sociedad plural.

c) Una metodología racional ${ }^{31)}$ de análisis de lo que hay en juego y del proceso. No se suele llegar a acuerdos por ensayo y error, ni bajo un falso criterio de la autoridad, sino que tiene su(s) propia(s) metodologías(s), que han de ser conocidas suficientemente por el clínico. Igual que no se diagnostica un linfoma por el mero ojo clínico, tampoco el proceso decisional se puede dejar al arbitrio de la intuición o de la experiencia particular de cada uno.

d) Diferencia entre una ética de mínimos y una ética de máximos. Nadie duda de la responsabilidad en respetar los mínimos morales, cuya transgresión nos sitúa debajo del umbral de la moralidad, lo que les convierte en innegociables. Un paciente, por ejemplo, no puede exigir a un médico, en función del principio de autonomía, que le ponga un tratamiento claramente contra- 
indicado. Ahora bien, para llegar a acuerdos necesitamos que se contemplen los proyectos de vida de las personas, es decir, su dimensión biográfica, que es la que construye los ideales de virtud o felicidad, la perspectiva de máximos. La vida va mucho más allá de la necesidad de regulación de las transaminasas.

e) Reconocimiento del otro como un interlocutor válido en condiciones de simetría moral, es decir, como un equal moral al que se le acepta porque es otro y no necesariamente por lo que piense. La gente respetable lo es toda, no sólo la que piensa como nosotros. Este reconocimiento nos permite acercarnos al otro como una riqueza (su realidad es mucho más rica que mi concepción sobre él), como una oportunidad y no como una amenaza. En la misma línea, ese reconocimiento para ser operativo nos demanda una actitud real de estar expuesto a los argumentos y la posición de los otros y dispuesto a aprender de los mismos. Reconocer al otro significa también asumir la diferencia y la complementariedad del ámbito de la indicación y del ámbito de la elección.

f) Actitud deliberativa, que conlleva el planteamiento de la interacción como un proceso racional y conjunto de búsqueda de la verdad multidireccional, que huye de toda comunicación unidireccional tipo "COnozco la verdad y tengo estrategias para comunicársela sin alterarle". Esta actitud deliberativa integra la paradoja de vivir con convicciones y estar abierto a la provisionalidad de las mismas. Sí a las convicciones; no a los dogmatismos.

Además de estas condiciones necesarias para poder llegar a acuerdos, conven- dría describir brevemente otras determinadas precondiciones deliberativas, realmente necesarias, aunque no suficientes, para que el profesional pudiera desarrollar el proceso de deliberación:

- Pregunta explícita por las emociones, fragilidades o prejuicios que me pueden estar impidiendo escuchar al otro, así como por los mecanismos de defensa propios y desadaptativos que también interfieren en esa escucha.

- Autorregulación emocional, compatible con un tono vehemente, que no impositivo, en la expresión de los argumentos.

- Aceptación de la incertidumbre y del ámbito de las probabilidades.

- Prudencia, entendida ésta como virtud intelectual que permite tomar decisiones racionales, o al menos razonables, en condiciones de incertidumbre.

- Esfuerzo por diferenciar los valores, las razones y las emociones en el proceso de deliberación. No olvidar que los sentimientos no tienen categoría moral, no son en sí ni buenos ni malos. La responsabilidad ética surge a la hora de plantearnos qué hacemos con ellos, si construir humanidad y vida o destruirlas.

- Aceptación de que toda confrontación sin acogida del mundo vital (emocional y axiológico) del otro es una agresión.

- Garantía de soporte, es decir, de que se seguirá apoyando, aunque la opción elegida por el paciente no coincida con la que el profesional sostiene. El apoyo, en determinados casos excepcionales, podrá ser vía derivación.

- Aceptación de un modelo de relación deliberativo (que no paternalista ni informativo), que utiliza la 
propuesta racional (y no la manipulación, ni la coacción) y la asertividad ( $\mathrm{y}$ no la agresividad ni la inhibición).

- Contar con estrategias comunicativas tipo "counselling", suficientemente bien entrenadas y supervisadas, garante técnico imprescindible. No hay que olvidar que aunque solemos -equivocadamente- encerrar los procesos de toma de decisiones en las salas de reuniones en las que hacemos sesiones clínicas en los Servicios (deliberación "in vitro"), donde normalmente se debería jugar el partido es delante del paciente, para poder realizar un auténtico proceso de TDC (deliberación "in vivo").

\section{PROTOCOLO DE ACOMPAÑAMIENTO AL PACIENTE EN EL PROCESO DE TOMA DE DECISIONES COMPARTIDAS}

Un proceso de TDC no es un momento único que se da en una conversación puntual. Comienza en el primer encuentro con el paciente y se va madurando en todo el recorrido asistencial. Parece importante poder explicitar en algún momento de ese proceso la presencia de las siguientes claves:

1. Ambiente de acogida, en el que desde el comienzo se aclara que se intentará ir haciendo conjuntamente el proceso de toma de decisiones, en el que iremos confrontando las distintas alternativas y en el que la actitud central será la de respeto a sus valores y a su ritmo.

2. Los procesos quedan facilitados cuando son realizados en contextos habilitantes, es decir, creando espacios que faciliten la tranquilidad y la menor presión emocional.

3. Mostrar disponibilidad y accesibilidad para volver a abordar las cuestiones en el caso de que sigan presentándose dudas, de modo que se facilite la expresión de las mismas de manera continua.

4. Explicitación de la garantía de soporte, es decir, que -como afirmábamos antes- decida lo que decida, le vamos a seguir apoyando en su proceso.

Sirviéndonos de los trabajos en el ámbito de la bioética clínica del profesor Gracia, ya referenciados, y de la reflexión y experiencia clínica de largos años, el protocolo que ahora presentamos intenta recoger una metodología que aunque, inicialmente, pueda parecer compleja, la práctica diaria nos dice que, una vez entrenada, ayuda enormemente al trabajo clínico. En la Tabla 3 aparecen escuetamente los pasos a dar, pasos que pasamos seguidamente a desarrollar:

\section{Tabla 3. Adaptado de Antequera et al. (29)}

Procedimiento de análisis de los conflictos, para la TDC
a) Aclaración de los hechos (tanto datos objetivos como subjetivos).
b) Identificación de los problemas que aparecen y elección del problema central.
c) Identificación de los valores en conflicto en el problema central.
d) Estudio de los cursos de acción posibles, tanto de los extremos como intermedios.
e) Identificación del (o de los) curso(s) óptimo(s) de acción.
f) Toma de decisiones compartida.
g) Pruebas de consistencia. 
1. Discusión de los aspectos técnicos. Es el momento de los hechos clínicos, el diagnóstico, el pronóstico y los tratamientos. Parece muy importante una buena clarificación de estos hechos, tanto médicos, como psicológicos o de otro tipo, tanto de los datos objetivos como de los subjetivos, porque los errores en esta materia los arrastraremos en los pasos ulteriores del análisis.

2. Identificación de los problemas que aparecen, para después poder jerarquizarlos; elección posterior del problema central sobre el que desarrollar los siguientes pasos. Una forma útil de enunciar los problemas es hacerlo en forma de preguntas.

3. Identificación de los valores en conflicto en el problema central del caso. Usamos el término valores por su gran amplitud y su máxima ambigüedad. Valores son todas aquellas cosas que son importantes para un ser humano y que nos exigen respeto. La vida es un valor, la salud también lo es, como asimismo el respeto a las creencias religiosas $\mathrm{O}$ las tradiciones de las personas. El lenguaje de los valores abarca otros muchos lenguajes como el de los derechos humanos, el de los cuatro principios de la bioética, etc. Para que haya conflicto, tiene que haber dos o más valores que nos exijan respeto y que se opongan entre sí, o que no sea posible tomar en consideración a la vez. En medicina es frecuente que uno de los valores en conflicto sea la vida y otro el bienestar del paciente, o la escasez de recursos, etc. Metodológicamente a veces es útil identificar una especie de tabla de valores en juego para, posteriormente, señalar cuáles de ellos entran en conflicto y cuáles no.
4. Estudio de los "cursos de acción" posibles, tanto de los extremos como de los intermedios. Los cursos extremos son los cursos a evitar, pues suelen transgredir uno de los valores en conflicto de manera muy clara. Los cursos intermedios pueden ser múltiples y su búsqueda conjunta puede ser enormemente creativa y rica.

5. Deliberación sobre el (los) curso(s) de acción óptimo(s). Pueden ser varios $y$, en ocasiones, secuenciales. Son aquéllos que lesionan menos los valores en conflicto.

6. Toma de Decisiones Compartida.

7. Pruebas de consistencia de la(s) decisión(es). Son muy útiles para comprobar, una vez tomada la decisión, la fortaleza de la misma, pues permite reafirmarnos en que no se ha perdido el rumbo. Serían estas tres:

- Prueba de la legalidad: ¿Es legal la decisión que va a tomar?

- Prueba de la publicidad: ¿Estaría dispuesto a defenderla públicamente?

- Prueba del tiempo: ¿Tomaría esta misma decisión si pudiera esperar algún tiempo más?

Cuando las decisiones tomadas están dentro del marco legal (donde se hospeda un porcentaje muy mayoritario de la población), cuando se estaría dispuesto a defenderlas en distintos foros, porque están sólidamente justificadas, y cuando no han sido tomadas bajo la presión de la urgencia, parece que es más fácil que pudiéramos calificar la decisión como prudente.

8. Redacción y firmas, si es el caso, de un Documento de TDC. Sabemos lo que cuesta en nuestro entorno dejar por escrito, firmar; sin embargo, lo escrito también genera compromisos; además, ofrece una determinada percepción de control y puede 
ayudar a la disminución de la culpa en los familiares si la decisión ha sido muy comprometida.

Nos parece de especial relevancia detenernos en la discusión de los puntos 4 y 5 , ya que consideramos que el proceso de deliberación habrá de tener algunas variables importantes, en lo que denominamos la "trama dialéctica", que hace referencia a la necesidad de tener en cuenta ambos polos de la variable, los cuáles no son necesariamente opuestos, pero sí están en íntima relación (ver Tabla 4) y, con mayor o menor peso, necesitan ser considerados. Los comentamos brevemente:

1. Dilema - Problema. Ya hemos comentado la necesidad de clarificar bien cuál es el territorio de la deliberación que, en la búsqueda de alternativas, intenta evitar los planteamientos dilemáticos de tipo sí/no, blanco/negro. Ello implica aceptar la complejidad y ampliar las perspectivas.

2. Otros Intervinientes - Afectados en el proceso de decisión. Parece muy

\section{Tabla 4. Conceptos útiles en trama dialéctica para un proceso de Toma de Decisiones Compartida}

1) Dilema - Problema.

2) Otros Intervinientes - Afectados en el proceso de decisión.

3) Indicación - Elección.

4) Riesgos - Beneficios.

5) Circunstancias - Consecuencias.

6) Contextos - Procesos.

7) Fines y Medios

8) Necesidades - Satisfactores.

9) Corto Plazo - Largo Plazo.

10) Emociones - Valores.

11) Urgente - Necesario.

12) Reversibilidad - Irreversibilidad. importante aclarar desde el principio quién quiere el paciente que le acompañen en el proceso de toma de decisiones, sea en el papel de consejeros, de representantes, etc., el que ellos definan. Es la mejor manera de evitar presiones indebidas y propiciar criterios y apoyos esperados, lo que favorece la percepción de control y también de prevención de posibles conflictos. Por otro lado, conocer quiénes son los posibles afectados en la toma de decisiones (stake holders) también será de utilidad. Si hay alguien en contra de determinadas decisiones, es importante saberlo para valorar en qué medida puede afectar al proceso.

3. Indicación - Elección. Es esencial tener claro que son dos ámbitos distintos, también con responsabilidades diferenciales y ambas forman parte intrínseca de la toma de decisiones, como hemos ya ido viendo y analizando. En nuestra experiencia, el elemento nuclear de la bioética clínica, al menos en los países del Primer Mundo, estriba en la dialéctica entre la indicación y la elección.

4. Riesgos - Beneficios. El balance de riesgos y beneficios no sólo se perfila frente a la salud biológica, sino también frente a la que podemos denominar como salud biográfica, es decir, la que tiene que ver con los proyectos vitales de las personas. Dicho de otro modo, ese balance no se articula teniendo sólo en cuenta los riesgos para la vida biológica o los beneficios para la supervivencia. Esta dialéctica también podía haberse presentado, en sentido estricto, entre quality of life y sanctity of life ${ }^{(32)}$.

5. Circunstancias - consecuencias. Toda toma de decisiones sin valo- 
ración de consecuencias de las distintas opciones es, cuanto menos, imprudente. La valoración de consecuencias es la que nos permitirá, cuando sea necesario, hacer excepciones a los principios, a lo deontológico, a lo normativo. Pongamos por caso: la sedación irreversible de un enfermo en situación avanzadaterminal tiene una parte de decisión que corresponde básicamente al sujeto enfermo. Puede haber algún caso, realmente particular, de negación pronóstica adaptativa en el paciente, que nos invita a pensar a los profesionales que plantearle explícitamente esa opción al paciente puede incrementar un sufrimiento significativo, claramente evitable e innecesario. Las consecuencias previsibles en el futuro y las circunstancias sujetas a determinados presentes son variables que ayudan a personalizar unas decisiones que, obviamente, no pueden ajustarse a ningún contenido material de protocolo. Este binomio también podría haber sido expresado como Normativo - Excepcional, porque las excepciones se suelen hacer en función de consecuencias graves y probables previstas.

6. Contextos - Procesos. La diferencia entre eficacia y efectividad nos ayuda a situar esta realidad. Un tratamiento eficaz puede ser efectivo en un contexto y no serlo en otro concreto. Si no tienes en cuenta las condiciones reales de las personas, habrá propuestas justificadas desde la medicina basada en la evidencia que realmente no van a funcionar. Lo mismo ocurre con la perspectiva de proceso y, más específicamente, de proceso de adaptación. Habrá momentos en que el paciente estará preparado para realizar una deli- beración determinada y habrá momentos en los que no será posible efectuarla, sencillamente porque se ve muy cerca del conflicto y ello le produce miedo, un miedo que puede anular a la persona.

7. Fines - Medios. Este binomio, de clara raigambre ética es central y, desafortunadamente, no siempre fácil de distinguir, por la tendencia humana de absolutizar determinadas mediaciones y, desde ahí, reconvertirlas en fines en sí mismos. Con un ejemplo lo veremos más claro. Cuando hay familiares de enfermos avanzados-terminales que montan la conocida como "Conspiración - Pacto de Silencio", en realidad presuponen que los clínicos tenemos un interés central en informar al paciente de todo; en primer lugar, porque es su derecho y, además, porque es la única manera de que sus decisiones puedan ser correctas. Es decir, conciben que la información es un fin en sí mismo. No lo entendemos así. El objetivo, el fin de la intervención con un paciente avanzado será el bienestar, el confort, la calidad de vida... y para ello utilizaremos mediaciones como los opiáceos, el apoyo emocional... y la información, que son claramente medios. Por ello mismo, al paciente que desee no ser informado se le respetará, porque muy probablemente el recorte o la ausencia de información le ayudará a su bienestar. Será muy difícil deliberar si existe a la base una confusión importante en la categorización fines-medios.

8. Satisfactores - Necesidades. Va en la misma línea, pero es útil su diferenciación. Por ejemplo, los elementos técnicos (sean diagnósticos, terapéuticos, etc.) son habitualmen- 
te satisfactores de determinadas necesidades, pero su consideración es equívoca. La disposición de los satisfactores no obliga a su utilización. Dicho de otro modo: no todo lo técnicamente posible es éticamente aceptable, porque no necesariamente responde a una necesidad. Por ejemplo, cuántas veces se utilizan pruebas diagnósticas (teóricos satisfactores de la necesidad de saber) cuyos resultados realmente no se van a tomar en cuenta para la toma de decisiones. La tempestad de movimientos -utilización de múltiples satisfactores-, por ejemplo, suele responder a la necesidad de resolver la angustia propia más que a identificar soluciones provisionales.

9. Corto Plazo - Largo Plazo. La temporalidad es una dimensión central en la experiencia humana. Las personas nos solemos mover más por los resultados inmediatos que por cualquier otra variable (por ejemplo, podemos encender un cigarrillo para disminuir la ansiedad, aunque conozcamos lo predisponente que puede ser el tabaco para el cáncer pulmonar). En cuanto a los resultados en salud esta dimensión parece básica, porque hay pacientes que no tienen largo plazo, pero lo buscan y lo desean y está, por tanto, en función de su posible negación, el hecho de que no quieran desprenderse del deseo.

10.Emociones - Valores. En todo proceso de deliberación sobre un tema importante surgirán las emociones. Será interesante identificar qué emociones están en el proceso y cómo están influyendo en el mismo. Por ejemplo, una emoción muy presente es el miedo, demasiado mal consejero para la toma de decisiones.
O la misma angustia. En la puerta de Urgencias de muchos hospitales se ve a hijos que intentan forzar el ingreso de un padre en situación terminal en la UVI y lo argumentan en que "mientras hay vida hay esperanza", cuando en realidad es la propia angustia lo que les lleva a plantear esa exigencia.

11.Urgente - Necesario. También las prisas son malas consejeras y trabajar desde la urgencia nos hace perder perspectiva. Un ejemplo habitual: la toma de decisiones tiene que ver también con las dinámicas preventivas, pero si no se delibera a fondo sobre ellas, dado que no son urgentes, los resultados a largo plazo pueden no ser lo beneficiosos que uno desearía.

\section{Reversibilidad - Irreversibilidad.}

Cuanto más irreversible sea una decisión, más ha de estar justificada. Este binomio no es habitual, pero su importancia es radical, dada la imposibilidad en corregir diferentes cursos de acción. La dinámica de lo temporal y reversible facilita estrategias de revaluación de resultados y posibilita corregir los errores.

Todos estos binomios pueden formularse, en función del caso, en forma de preguntas, que pueden ser claves para el proceso deliberativo. De hecho, en muchas ocasiones es útil que el propio profesional sugiera al paciente formularse algunas preguntas importantes. A modo de ejemplo: ¿Quiénes pueden verse realmente afectados por esta decisión que estamos valorando?, ¿qué beneficios esperamos de esta alternativa, qué riesgos asumimos con ella?, ¿qué consecuencias podrían tener cada una de estas decisiones?, ¿qué carga emocional implica el tomar esta decisión?, etc. 


\section{A PROPÓSITO DE UN CASO DE TOMA DE DECISIONES COMPARTIDA EN UN PROCESO DE LIMITACIÓN DEL ESFUERZO TERAPÉUTICO}

Motivo de consulta: EAJ es una mujer de 18 años de edad, con incapacidad jurídica, que es remitida al Servicio de Neumología desde la Reanimación de Traumatología de un gran hospital público, donde estuvo ingresada durante 5 meses tras cirugía de artrodesis de columna.

Antecedentes personales significativos: microcefalia congénita, encefalopatía grave, crisis comiciales, múltiples malformaciones (polidactilia, hipertrofia de labios menores, escoliosis). Incontinente. No se relaciona con el medio.

Tratamiento habitual: Lexatin 1,5 al día; Luminal 0,1 al día; Noctamid por la noche.

Enfermedad actual: la paciente fue intervenida 5 meses antes de artrodesis vertebral posterior con fijación sacra para corrección de escoliosis. Se consigue extubar 24 h. después de la cirugía. No obstante, dos días más tarde presenta un cuadro de insuficiencia respiratoria aguda grave, se ingresa en la Reanimación y recibe una intubación oro-traqueal.

En la Reanimación presenta múltiples infecciones respiratorias por $P$. aeruginosa multirresistente así como dificultad para expectorar y para el destete. Desde que tiene la traqueostomía se han manifestado desaturaciones y crisis comiciales.

Posteriormente se estabiliza y se pauta seguimiento neurológico y sonda nasogástrica para nutrición. Se pasa a sedestación en una silla especial. Desde el punto de vista respiratorio aparece una colonización por $P$. aeruginosa; mantiene unas buenas saturaciones con oxígeno y ventilación mecánica.

Se pasa a la planta de Neumología, donde tienen conocimiento previo del caso, tanto de la enferma como de la familia. Se realiza colocación de ventilación mecánica no invasiva (VMNI) tipo BiPAP a cánula de traqueostomía. A los tres días de estancia aparece una fuerte desaturación y sepsis. Por primera vez se habla a fondo con los padres, comentando el estado de la hija y sus posibilidades. Ante el estado de la paciente y las posibilidades expuestas, la madre nos preguntó: ¿Qué haría usted? Se le expusieron dos escenarios: en la Reanimación habría conexión a ventilación mecánica, mayor riesgo de infecciones, menor posibilidad de visitas y una posible supervivencia más corta; en la planta de Neumología, más o menos igual supervivencia, una presencia constante de la familia, una conexión similar a ventilación mecánica y un tratamiento de las complicaciones intercurrentes que no requirieran medidas extraordinarias. La madre volvió a repetir la pregunta y se le contesta: Creemos que por todo lo dicho estará mejor con nosotros, en la planta. Si empeora y cambian de opinión siempre podemos llamar a la Reanimación. Consultó con su marido y se decidió que permaneciese en la planta de Neumología. Se constató un cambio de relación con la familia a partir de consultarles cambios de actitud en los cuidados de la paciente y posibilitando una relación más cercana. A partir del cambio de actitud se empezó a plantear decisiones de proceso a tomar con su hija. Se consulta con el equipo de psicólogos de Hematología (Neumología no dispone de ese recurso), se van tomando decisiones y se realiza por escrito un documento de TDC que se firma. Exponemos el documento, aclarando en la columna de la derecha la perspectiva de fondo de los distintos párrafos.

Los abajo firmantes... EXPONEN las siguientes consideraciones: 


\begin{tabular}{|c|c|}
\hline $\begin{array}{l}\text { Después de haber deliberado sobre el estado de salud } \\
\text { de EAJ y la compleja situación actual, todos compar- } \\
\text { timos el objetivo y el deseo de priorizar su calidad de } \\
\text { vida y de que no sufra innecesariamente. }\end{array}$ & $\begin{array}{l}\text { - Proceso: deliberación. } \\
\text { - Objetivos: CV }+\downarrow \text { Sufri- } \\
\text { miento }\end{array}$ \\
\hline $\begin{array}{l}\text { Queremos hacer este camino de decisiones juntos, } \\
\text { porque entendemos que la toma de decisiones com- } \\
\text { partida disminuye el riesgo de equivocarnos, al tener } \\
\text { tanto en cuenta la dimensión médico-sanitaria de } \\
\text { EAJ, como su dimensión biográfica, conocida sobra- } \\
\text { damente por sus padres. Todo ello es compatible con } \\
\text { la afirmación de que los principales responsables de } \\
\text { EAJ son sus padres, pero que los profesionales quie- } \\
\text { ren corresponsabilizarse de este proceso para apoyar } \\
\text { tanto a la paciente como a su familia. }\end{array}$ & $\begin{array}{l}\text { - Voluntad de decisiones } \\
\text { compartidas. } \\
\text { - Corrección del riesgo. } \\
\text { - Dimensión biológica y bio- } \\
\text { gráfica. } \\
\text { - Responsabilidad de los pa- } \\
\text { dres y corresponsabilidad } \\
\text { de los profesionales. Garan- } \\
\text { tía de soporte. }\end{array}$ \\
\hline $\begin{array}{l}\text { Nuestro deseo es preservar la } \\
\text { pertenece por el hecho de ser }\end{array}$ & $\begin{array}{l}\text { - Reconocimiento explícito } \\
\text { de la dignidad de la pacien- } \\
\text { te. }\end{array}$ \\
\hline $\begin{array}{l}\text { Entendemos que los criterios de calidad de vida para } \\
\text { EAJ se centran en que pueda vivir en su hogar de } \\
X X X X X X(X X X) \text {, en compañía de su familia, más es- } \\
\text { pecíficamente de sus padres, hermanos y abuelos. } \\
\text { Ellos son los que pueden aportarle el bienestar que } \\
\text { EAJ necesita. }\end{array}$ & $\begin{array}{l}\text { - Concreción de CV / bienes- } \\
\text { tar: vivir con su familia. } \\
\text { - Reconocimiento del papel } \\
\text { beneficente de la familia. }\end{array}$ \\
\hline $\begin{array}{l}\text { Apreciamos que la vida de EAJ ha sido vivida como } \\
\text { un regalo para su entorno familiar, que la concibe } \\
\text { como "un ángel del cielo, una sonrisa y una mirada } \\
\text { permanente". }\end{array}$ & $\begin{array}{l}\text { - Reconocimiento de la apor- } \\
\text { tación de la paciente a su } \\
\text { entorno. }\end{array}$ \\
\hline $\begin{array}{l}\text { Tanto la médico como el psicólogo quieren explicitar } \\
\text { que los cuidados y las atenciones de los padres a EAJ } \\
\text { están siendo impecables y valoramos enormemente } \\
\text { el cuidado práctico y afectivo que otorgan a su hija. } \\
\text { Sin duda alguna, sin estos cuidados difícilmente EAJ } \\
\text { hubiera podido llegar a la edad que ahora tiene. }\end{array}$ & $\begin{array}{l}\text { - Reconocimiento del papel } \\
\text { cuidador de los padres, en } \\
\text { su vertiente práctica y afec- } \\
\text { tiva. }\end{array}$ \\
\hline $\begin{array}{l}\text { También los profesionales queremos hacer constar } \\
\text { que los padres han tomado siempre las decisiones } \\
\text { más convenientes a la luz de los datos disponibles en } \\
\text { cada momento. Si las cosas no han ido como todos } \\
\text { hubiéramos deseado no ha sido por una conducta } \\
\text { imprudente, sino porque las consecuencias de las } \\
\text { decisiones no pueden ser siempre predecibles. }\end{array}$ & $\begin{array}{l}\text { - Reconocimiento de la va- } \\
\text { lidez de las decisiones to- } \\
\text { madas previamente por los } \\
\text { padres. } \\
\text { - Prevención de futura culpa. }\end{array}$ \\
\hline
\end{tabular}


(Todos los reconocimientos pretenden un encuadre terapéutico, la facilitación de la posterior toma de decisiones y la prevención de un posible duelo complicado).

A la luz de estas consideraciones, ACORDAMOS ADOPTAR LAS SIGUIENTES DECISIONES:

( Rechazar un nuevo ingreso en la Unidad de Cuidados Intensivos.

- Rechazar la implantación de un respirador para mantener la función respiratoria.

- Rechazar cualquier tipo de medidas especiales extraordinarias, como puede ser, a modo de ejemplo, la diálisis.

( Si tuviera una parada cardiorrespiratoria fácilmente reanimable, estaríamos de acuerdo en que se la intentara remontar, siempre y cuando ello le permitiera poder seguir ingresada en la planta. Si precisara el ingreso en la Unidad de Cuidados Intensivos, rechazamos la reanimación cardipulmonar.

- Aceptamos que le hagan una gastrostomía para facilitar la nutrición.

Aceptamos la sedación profunda e irreversible si no hay otra posibilidad para controlar determinados síntomas que generen sufrimiento a EAJ.

(1) Solicitamos que se le provea de aquellos cuidados y tratamientos ordinarios que respondan a problemas de salud habituales y que no sean ni muy invasivos ni muy molestos para EAJ.

Rechazamos de plano la eutanasia activa y directa para EAJ.
Somos conscientes de que la salud de EAJ está en un momento crítico y que si llegara el doloroso momento de su muerte, ésta no habría sido producida por las decisiones tomadas, sino por el proceso natural y las complicaciones de sus distintas enfermedades.

La médico y el psicólogo quieren agradecer a los padres la valentía para hablar de estas cosas, pues aun sabiendo que el hablar y el decidir clarifica enormemente la tarea de los profesionales y las decisiones de los padres a la hora de perseguir el bienestar de EAJ, no por ello deja de ser doloroso para estos últimos el abordar estas cuestiones.

Este documento puede ser revocado por los padres en cualquier momento.

Firmas de los padres y profesionales.
- Explicación de la posible causa de muerte. Prevención de duelo complicado.

- Agradecimiento a los padres por la valentía a la hora de afrontar las dificultades.
- Posibilidad de revocación.

- Firmas de padres y profesionales.
Al mes de firmarse el documento, la paciente entra en crisis con una insuficiencia respiratoria grave. Se aplican tratamientos ordinarios, incluyendo la colocación de la VMNI, y no se resuelve la crisis. Se avisa al padre, que trabajaba fuera de la ciudad, aportando mientras tanto medicación para alivio de los síntomas respiratorios. La paciente presenta cada vez más signos de molestia y de inquietud y se acuerda con los padres, ante la no respuesta terapéutica y ante los acuerdos firmados, la sedación 
profunda e irreversible. La paciente fallece a las 36 h. Los padres, con el dolor propio de la situación, manifiestan la tranquilidad de haber hecho lo que tocaba y estaba consensuado, en función de los valores que había en juego y no en función de la angustia que inevitablemente podía acompañar a la situación y a las decisiones.

El concepto de TDC no remite únicamente al vínculo paciente (representante) - profesional responsable del seguimiento, sino también a compartir decisiones entre profesionales y entre Servicios clínicos. En el caso presentado el proceso se gestionó profesionalmente entre la médico pneumóloga y el psicólogo; en otros muchos casos se desarrolla entre el Servicio implicado y el Equipo de CP, etc. Este vínculo interdisciplinar e inter-Servicios facilita enormemente estos procesos; no sólo porque amplíe el campo de mira, sino también porque ayuda a cotejar opiniones, a disminuir el riesgo de pérdida de horizonte por cuestiones emocionales (por ejemplo, relación ya muy cargada), a compensar tensiones y a objetivar la indicación clínica.

\section{ÚLTIMAS REFLEXIONES}

a) Se ha hablado en todo este trabajo de lo que supone un proceso de TDC. Ahora bien, en definitiva, ¿Quién acaba tomando las decisiones $y$-fundamentalmente- las más críticas? La experiencia nos dice que las podemos tomar conjuntamente si en la deliberación realmente conjugamos la realidad y el deseo, los hechos y los valores, las razones y las emociones; si además nos reconocemos realmente como interlocutores válidos en condiciones de simetría moral y si, por último, somos capaces de no huir los unos de los otros. Una manera de huir es ir tomando nosotros habitualmente las decisiones, excepto cuando hay un conflicto importante, que entonces le decimos al otro que ejerza él desde su maravillosa autonomía.
Conviene señalar que, en la estructura vincular, existen un protagonista (el paciente o su representante) y un coprotagonista (el profesional) y en su funcionalidad el "yo" del paciente no se sitúa frente al "tú" del profesional o en sentido inverso, sino que mutuamente deliberan para conjugar en clave de primera persona del plural, en clave de un "nosotros", a sabiendas que el procedimiento es conjunto aunque la última decisión, de no haber consenso y si no se nos exige nada realmente maleficente o injusto, acaba siendo del paciente. Es otra manera de entender el principio de autonomía. El proceso conjunto, común, compartido de toma de decisiones integra la dinámica de la elección y de la indicación, sin olvidarse de que de lo que estamos hablando, fundamentalmente, es de la salud, la enfermedad, la vida y la muerte del propio paciente.

b) ¿Qué tipo de relación se establece en ese proceso de TDC? Una relación deliberativa -ni paternalista ni meramente informativa- que supone vínculo y que conlleva compromiso. El compromiso va más allá que la implicación. De hecho los adjetivos son importantes: solemos hablar de implicación emocional y de compromiso moral. En la TDC hay un claro compromiso ético con la realidad global de la persona (tanto biológica como biográfica), lo que supone trabajar el proceso desde una dinámica específica de acompañamiento. Deliberación y acompañamiento tienen mucho en común. Puede haber acompañamiento sin deliberación, si es que en el itinerario de acompañamiento no se entra en el ámbito de la toma de decisiones; sin embargo, no puede haber auténtica deliberación acerca de lo que hay en juego para una decisión si no se realiza desde una actitud de acompañamiento. La persona puede tener dudas, sensación de indefensión, incertidumbre... y necesitar un compañero de viaje que, además de ayudarle a discernir entre va- 
lores, medios y fines, etc., le sostenga en ese viaje, sobre todo en los momentos de más dificultad. Acompañar, en el terreno de la enfermedad, se exige para deliberar, porque exige sostener, si toca, en medio de la desolación.

c) Participar en un proceso de TDC, ¿no supone una cierta sobreimplicación para el profesional? No necesariamente. En primer lugar, en el proceso de deliberación te encuentras con datos positivos y relevantes y con valores de la persona que nos permite contrabalancear los datos más preocupantes, problemáticos y amenazantes, lo que favorece el equilibrio emocional para la relación. Podemos decir que el médico -u otro profesionalque delibera se agota (supone asumir la intensidad del vínculo), pero no se quema, porque la riqueza recibida en la deliberación compensa las frustraciones vividas; de hecho, la experiencia es que el encuentro deliberativo se convierte en un proceso y escenario de claro aprendizaje mutuo. Además, el trabajo de una TDC permite que la persona enferma perciba que a ti, profesional, te importa lo que a él le importa (porque lo acoges en la deliberación), lo que fortalece un vínculo de confianza, tolerancia y compromiso, los mejores antídotos contra el miedo.

La incertidumbre y el miedo suelen estar presentes en todo proceso de toma de decisiones. Precisamente, conocerlo y ser conscientes de ello, paradójicamente da control. Los propios pacientes nos reflejan que en la propia deliberación, gracias a la actitud de acogida perciben menos amenaza en medio de la incertidumbre y pueden, desde la confianza, descansar en los momentos de mayor inseguridad.

Promover y participar en procesos de TDC es todo un desafío para los profesionales. Nos hace ver que para lograr una buena comunicación y deliberación con el otro necesitamos alimentar una adecuada introspección y discernimiento hacia uno mismo, lo que evitará estar proyectando nuestras dificultades y nuestras posiciones en el que tenemos en frente. Enriquecer esta perspectiva de TDC nos invita a promover el diálogo frente a la imposición, la explicitación frente a los supuestos, la confianza frente a la sospecha, la consideración del otro primeramente como persona-ciudadano que no como enfermo-in/ firme. En definitiva, una llamada a aceptar el reto que supone todo encuentro significativo (está la vida y la calidad de vida en juego), entre personas.

Llamamos a la reflexión acerca de la necesidad de identificación del tipo de proceso(s) preponderante(s) de toma de decisiones que utilizamos, de forma consciente o no, en nuestros Servicios asistenciales. Tienen detrás un modelo. Tomar conciencia de ello es el primer paso para promover mejoras. A partir de ahí, puede ser interesante poner en marcha iniciativas que nos ayuden a profundizar en esta línea. Una de ellas, en algunos Servicios, han sido los programas de elaboración de Instrucciones Previas ${ }^{(33),}$ en los que el proceso acaba siendo al menos tan interesante como el mismo resultado. Ya ven qué cosas, compartir un proceso de toma de decisiones, correcta y conscientemente, es la mejor manera de reivindicar el derecho a la autonomía moral de la persona. Un buen guionista podrá plasmar la película de su propia vida si cuenta con un buen equipo de producción que le ayude a que su proyecto no quede tan solo en el mundo de las ideas o de las buenas intenciones. El productor peleará para que ese film represente fielmente la letra y la música, el alma y el corazón de lo que el guionista autobiográfico ha destacado como propio... y el autor del guión contará siempre con el productor para que le facilite la más bella realización de su deseo. 


\section{REFERENCIAS BIBLIOGRÁFICAS}

1. Barbero J, Romeo C, Gijón P, Júdez J. Limitación del esfuerzo terapéutico. En: Gracia D, Júdez J, editores. Ética en la práctica clínica. Madrid: Fundación Ciencias de la Salud / Triacastela, 2004; 161-81.

2. O'Connor A, Stacey D, Entwistle $V$ et al. Decision aids for people facing health treatment or screening decisions. Cochrane Database Syst Rev: CD001431, 2003. Doi: 10.1200/JCO.2010.29.9768.

3. President's Commission for the Study of Ethical Problems in Medicine and Biomedical and Behavioral Research. Making Health Care Decisions. The ethical and legal implications of informed consent in the patient-practitioner relationship. Washington, 1982.

4. Charles C, Gafni A, Whelan T. Shared decision-making in the medical encounter: what does it mean? (Or it takes at least two to tango). Soc Sci Med 1997; 44 (5): 65161.

5. Charles C, Gafni A, Whelan T. Decisionmaking in the physician-patient encounter: Revisting the shared treatment decisionmaking model. Soc Sci Med 1999; 49: 681-92.

6. Kasper J, Lenz M. Criteria for the development and evaluation of decision aids. Z Artl Fortbild Qualitatssich 2005; 99: 359-65.

7. Makoul G, Clayman M. An integrated model of shared decision making in medical encounters. Patient Educ Couns 2006; 60 (3): 301-12. Doi: 10.1016/j. pec.2005.06.010.

8. Towle, A, Godolphin W. Framework for teaching and learning informed shared decision making. Br Med J 1999; 319(7212):766-771. Doi: 10.1016/j. pec.2005.06.010.

9. Braddock C, Edwards K, Haseng N, Laidley $\mathrm{T}$, Lewinson W. Informed decision making in outpatient practice: time to get back to basics. JAMA 1999; 282: 2313-20. Doi: 10.1001/jama.282.24.2313.

10. Taylor S, Pickens J, Geden E. Interactional styles of nurse practitioners and physicians regarding patient decision making. Nurs Res 1989; 38:50-5.

11. Jordan J, Ellis S, Chambers R. Defining shared decision making and concordance: are they one and the same? Postgrad Med J 2002; 78: 383-4.

12. Ruland C, Bakken S. Developing, implementing, and evaluating decision support systems for shared decision making in patient care: A conceptual model and case illustration. J Biomed Inform 2002; 35:313-21.

13. Ford S, Schofield T, Hope T. What are the ingredients for a successful evidence-based patient choice consultation? A qualitative study. Soc Sci Med 2003; 56: 589-602. Doi: 10.1016/S0277-9536(02)00056-4.

14. Stubblefield C, Mutha S. Provider-patient roles in chronic disease management. J Allied Health 2002;31: 87-92.

15. Gracia D. La deliberación moral: el método de la ética clínica. Med Clin (Barc) 2001; 117: 18-23.

16. Emanuel EJ, Emanuel LL. Four Models of the Physician-Patient Relationship. JAMA 1992; 267: 2221-2226. (Trad. cast: Cuatro modelos de la relación médico-paciente. En: Couceiro A, editor. Bioética para clínicos. Madrid: Triacastela, 1999. p. 109-26.

17. Arranz P, Barbero J, Barreto P, Bayés R. Intervención emocional en Cuidados Paliativos. Modelo y protocolos. Barcelona: Ariel, 2003.

18. Kasper J, Geiger F, Freiberger, S and Schmidt A. Decision-related uncertainties perceived by people with Cancer modelling the subject of shared decision making. Psychooncology 2008, 17, 42-8. Doi: 10.1002/pon.1190.

19. Harrison TR, Fauci A, Brawnwald E, Kasper DL. Principios de Medicina Interna, 14 edición. Madrid: McGraw-Hill / Interamericana de España, S.A, 1998. 
20. Ley 41/2002, básica reguladora de la autonomía del paciente y de derechos y obligaciones en materia de información y documentación clínica. BOE núm 274, de 15 de noviembre de 2002. p. 40126-32.

21. Barbero J. Paciente no competente $y$ capacidad de elección. Jano 2008 (21-27 nov); 1717: 33-5.

22. Barbero J. El derecho del paciente a la información: el arte de comunicar. An Sist Sanit Navar 2006; 29, 3: 19-27.

23. Beauchamp TL, Childress JF. Principios de Ética Biomédica. Barcelona: Masson, 1999. (Traducción de la $4^{a}$ edición).

24. Centeno C, Núñez Olarte JM. Estudios sobre la comunicación del diagnóstico de cáncer en España. Med Clin (Barc) 1998; 110: 744-50.

25. Simón $P$, Domenech $P$, Sánchez Beiza $L$, Blanco T, Frutos B, Barrio I. ¿Querría usted saber que tiene cáncer? Med Fam 1994; 4, 3: 127-36.

26. Díaz-Sayas L, Cruzado JA, Barbero J. Estudio de las micro-malas noticias en pacientes hematológicos hospitalizados. La comunicación médica diaria. Psicooncología 2010; 7,1: 175-91.
27. Barbero J. Psicólogos en Cuidados Paliativos: El largo camino de la extrañeza a la Integración. Clin Contemp 2010; 1, 1: 39-48.

28. Gracia D. Moral Deliberation. The role of methodologies in clinical ethics. Med Health Care Philos 2001; 4:223-32. Doi: 10.1023/A:1011445128427.

29. Antequera JM, Barbero J, Bátiz J. et al. Ética en cuidados paliativos. Guías de ética en la práctica médica - 2. Madrid: Fundación de Ciencias de la Salud, 2006.

30. Gracia D. Fundamentos de Bioética. Madrid: Eudema Universidad, 1989.

31. Gracia D. Procedimientos de Decisión en Ética Clínica. Madrid: Eudema, 1991.

32. Barbero J. Calidad de vida guiada por los valores. Reflexiones desde una Casa de Acogida para Enfermos de Sida. En: Quaderni di Bioètica e Cultura 2: The Sanctity of Life. Roma 1994; 139-49.

33. Barbero J, Díaz L, Coca C, del Rincón C, López-Fando T. Las Instrucciones Previas en funcionamiento: una herramienta legal con valor psicoterapéutico. Psicooncología 2008; 5, 1: 117-27. 